\title{
Uma Revisão Sistemática de Variáveis Relevantes na Produção de Erros em Matemática
}

\section{A Systematic Review of Relevant Variables in the Production of Errors in Mathematics}

\author{
Gabriele Gris* \\ ORCID iD 0000-0002-7755-9430 \\ Livia dos Santos Palombarini** \\ ORCID iD 0000-0002-5981-0994 \\ João dos Santos Carmo*** \\ ORCID iD 0000-0003-3913-7023
}

\begin{abstract}
Resumo
Questões envolvendo a identificação, análise e interpretação de erros cometidos por estudantes em Matemática não são recentes, embora muito ainda possa ser investigado sobre esse assunto. $\mathrm{O}$ objetivo do presente estudo foi identificar, a partir da literatura já existente, variáveis relevantes na produção de erros em Matemática. Uma revisão sistemática da literatura do período entre 2012 e 2017 foi realizada, de forma independente, por duas pesquisadoras para avaliar a concordância entre elas. Foram pesquisados artigos nas bases de dados ERIC, PsycArticles, SciELO e Math Educ Database com os descritores erro AND matemática; erro AND procedimento AND matemática; padrão de erro AND matemática; análise de erros AND matemática; erro sistemático AND matemática e seus correspondentes em inglês e espanhol. Foram identificados 415 artigos, dos quais foram analisados 31 que tratavam da produção de erro. As variáveis identificadas como responsáveis pela produção dos erros mais comuns referem-se às causas internas ou dificuldades não especificadas do aluno e aos erros nos procedimentos de ensino. A responsabilidade pelo erro geralmente é atribuída aos alunos e a principal tendência da pesquisa é apenas informar a produção de erros, uma vez que apenas alguns estudos indicaram formas de evitar ou lidar com os erros produzidos pelos alunos de maneira específica e descritiva. Ressalta-se a importância e a necessidade de investigar práticas educativas para prevenir e lidar com erros.
\end{abstract}

Palavras-chave: Padrões de Erro. Produção de Erros. Categorização de Erros. Ensino de Matemática.

\begin{abstract}
* Mestre em Análise do Comportamento (UEL). Doutoranda em Psicologia da Universidade Federal de São Carlos (UFSCar), membro do Instituto Nacional de Ciência e Tecnologia sobre Comportamento Cognição e Ensino. Departamento de Psicologia, Universidade Federal de São Carlos, Rodovia Washington Luis, Km 235, SP 310, Bairro Monjolinho, Cep 13565-905 E-mail: ggris@ ufscar.br.

${ }^{* *}$ Licenciada em Física (UFSCar). Mestre em Psicologia da Universidade Federal de São Carlos (UFSCar), membro do Instituto Nacional de Ciência e Tecnologia sobre Comportamento Cognição e Ensino. Departamento de Psicologia, Universidade Federal de São Carlos, Rodovia Washington Luis, Km 235, SP 310, Bairro Monjolinho, Cep 13565-905 E-mail: liviapalombarini@ufscar.br.

**** Doutor em Educação (UFSCar). Professor Adjunto da Universidade Federal de São Carlos (UFSCar), membro do Instituto Nacional de Ciência e Tecnologia sobre Comportamento Cognição e Ensino. Departamento de Psicologia, Universidade Federal de São Carlos, Rodovia Washington Luis, Km 235, SP 310, Bairro Monjolinho, Cep 13565-905. E-mail: jcarmo@ufscar.br.
\end{abstract}


Issues involving the identification, analysis, and interpretation of mistakes made by mathematics students are not recent, although much still can be investigated on this subject. The aim of the present study was to identify, from the existing literature, relevant variables in the production of errors in mathematics. A systematic review of the literature of the period between 2012 and 2017 was independently performed by two researchers to evaluate the concordance between them. We searched the ERIC, PsycArticles, SciELO and Math Educ Database databases with the descriptors error AND mathematics OR math, error AND procedure AND mathematics OR math, error pattern AND mathematics OR math, analysis of errors AND mathematics OR math, systemic error AND mathematics OR math and their correspondents in Portuguese and Spanish. A total of 415 articles were identified, of which 31 were analyzed, dealing with error production. The variables identified as responsible for producing the most common errors refer to the student's internal causes or unspecified difficulties and errors in the teaching procedures. Responsibility for error is usually attributed to the students and the main trend of the research is only to inform the production of errors, since only a few studies have indicated ways to avoid or deal with errors produced by students in a specific and descriptive way. We emphasize importance and necessity of investigating educational practices to prevent and deal with errors.

Keywords: Error Patterns. Error Production. Categorization of Errors. Mathematics Teaching.

\section{Introdução}

Estudos sobre produção, interpretação e análise de erros de estudantes são realizados há várias décadas, embora diversas questões ainda possam ser investigadas com vistas à produção de conhecimento que possibilite novas práticas pedagógicas. A associação entre erro e fracasso do estudante é bastante comum no ambiente escolar e tende a confirmar formas de classificar "bons" e "maus" alunos com base em desempenhos avaliados como corretos ou incorretos (CARVALHO, 1997). Apesar dessa associação corriqueira, há por parte de muitos pesquisadores e educadores entendimentos diversos sobre o que é um erro e qual é a sua função.

Em uma revisão de literatura em periódicos brasileiros de Educação Matemática, Silva, Salvi e Passos (2013) identificaram, por meio de análise de conteúdo, unidades de registro e categorias para reunir interpretações sobre o erro em publicações até o ano de 2010. Os autores destacam categorizações dos erros enquanto reveladores, diagnosticadores, mobilizadores, essenciais/intrínsecos, fecundos e lógicos. Tais categorias parecem qualificar erros positivamente, explorando possibilidades pedagógicas a partir de sua ocorrência, como defendido em outros trabalhos da área (ALMEIDA; PIZANESCHI; DARSIE, 2016; SPINILLO et al., 2014)

Carmo (2010) também questiona a prática de tratar erros e acertos como "tudo ou nada", destacando que esse tipo de classificação pode não identificar todas as variáveis envolvidas na questão. $\mathrm{O}$ autor, entretanto, operacionaliza o erro em contexto escolar evitando caracterizá-lo positiva ou negativamente. O erro é descrito simplesmente como "um padrão de resposta que não atingiu determinado critério que permita a aprovação por parte do professor 
e/ou a passagem para outra etapa de aprendizagem" (CARMO, 2010, p. 214). Tal definição é semelhante à perspectiva adotada por Moroz (2012) que descreve o erro como um repertório que não alcançou um padrão esperado, uma vez que o ensino não produziu o aprendizado requerido para determinada situação.

Ao admitir que o significado do termo erro é amplo, percebe-se a importância de investigá-lo sob diversos pontos de vista (CARMO, 2010). Há pelo menos duas questões que têm recebido destaque na literatura quanto à produção de erros em Matemática: uma sobre a identificação de padrões de erros e outra sobre as diversas fontes de erros no contexto escolar. A discussão de ambas está relacionada e a opção pela separação tende a ser apenas didática.

Diante da diversidade dos estudos que tratam sobre a produção de erros em Matemática, destaca-se a importância de reunir algumas informações para efeito de análises mais detalhadas. De acordo com Von Hohendorff (2014), uma revisão sistemática de literatura visa sumarizar pesquisas prévias para responder questões e reunir evidências. Assim, o objetivo do presente estudo foi identificar, a partir da literatura já existente, variáveis relevantes na produção de erros em Matemática. Com base nos resultados buscou-se discutir as seguintes questões: que tipos de variáveis levam ao erro? Quais as principais tendências dos estudos quanto à categorização de erros? Para tanto, inicialmente são apresentados panoramas gerais da literatura sobre padrões de erros em Matemática e possíveis fontes desses erros. Posteriormente apresenta-se a descrição da revisão sistemática, seus resultados e discussões relacionadas à literatura previamente apresentada.

\subsection{Padrões de erros em Matemática}

O conhecimento matemático para o ensino deve englobar também a forma como os professores lidam com os erros apresentados pelos alunos (CURY, 2013). Desde a década de 1970, há consistentes produções sobre padrões de erros em cálculo nos trabalhos de Ashlock (1998) que buscou detectar erros sistematicamente produzidos por estudantes em tarefas de Matemática. Mesmo estudos anteriores aos do autor já buscavam identificar e classificar os erros produzidos por estudantes (ARTHUR, 1950; GROSSNICKLE, 1936; ROBERTS, 1968). O estudo de Grossnickle (1936), por exemplo, analisou os erros e os "hábitos questionáveis" em atividades de divisão por meio de 453 avaliações do $5^{\circ}$ ao $8^{\circ}$ ano. Os erros foram agrupados em sete categorias: erros de combinação, erros resultantes do uso do resto, erros na presença do zero, erros de procedimentos falhos, erros por lapsos de atenção, erros causados ao "abaixar" números, erros por falhas ou hábitos questionáveis. 
Com o objetivo de identificar as habilidades de cálculo em que os estudantes apresentavam maiores dificuldades, o estudo de Roberts (1968) analisou os desempenhos de 148 estudantes da terceira série de escolas públicas de Nova York. O autor relatou encontrar 57 tipos de erros que foram categorizados em: operações erradas, erros de cálculo óbvios, algoritmos incompletos/incorretos e respostas aleatórias. Além de categorizar os erros, o autor apresentou a frequência em cada categoria, breves interpretações sobre os tipos de erros e sugestões para melhorar o desempenho dos estudantes a partir dos erros produzidos.

Alguns estudos, além de categorizar os erros, buscaram avaliar procedimentos de correção. Stefanich e Rokusek (1992) analisaram os erros produzidos pelos estudantes da $4^{\mathrm{a}}$ série no uso do algoritmo de divisão com números inteiros e investigaram os efeitos de um procedimento de correção sobre os erros sistemáticos identificados. Participaram do estudo 25 estudantes, aleatoriamente selecionados de uma população de 47 estudantes.

Inicialmente foi aplicado um instrumento de diagnóstico e analisou-se os padrões de erros nas atividades. Os erros foram classificados em: descuido, aleatórios, sistemáticos e de omissão. Foram identificados erros sistemáticos nas atividades de 11 estudantes que receberam dois dias de instruções de correção. No primeiro dia foi revisado o conceito de algoritmo e criado um procedimento de cinco etapas a serem seguidas na resolução de problemas.

No segundo dia foi realizada uma revisão geral do material abordado no primeiro dia. Cada tipo de erro foi apresentado e exemplos específicos dos testes diagnósticos das crianças foram usados. Os alunos receberam instrução direta envolvendo a correção desses erros e foram então solicitados a identificar e corrigir erros semelhantes para enfatizar o reconhecimento dos mesmos. Posteriormente, todos os estudantes realizaram o mesmo teste utilizado inicialmente para diagnosticar os padrões de erros.

De modo geral, a partir da comparação dos resultados dos testes feitos, observou-se redução dos erros cometidos após a intervenção em todas as categorias. Foram identificados, por exemplo, 56 erros sistemáticos na primeira avaliação e oito após os procedimentos de correção.

Para analisar os padrões de erro na resolução de operações de adição, subtração, multiplicação e divisão, Bourke (1979) descreveu os erros de 15 estudantes do $5^{\circ}$ ano em categorias como método ineficiente, erro de algoritmo, sem resposta, erro por "descuido", erro de cálculo, erro de codificação, ausência de compreensão conceitual da operação e motivação. Foram identificados maior número de erros decorrentes de métodos ineficientes e erros de algoritmos. 
Uma das principais contribuições metodológicas do estudo está relacionada à seleção dos participantes. Ao considerar que um erro pode ser influenciado por diferentes variáveis, o autor selecionou participantes que apresentavam dificuldades na resolução de operações, mas que apresentavam repertórios competentes de leitura com compreensão das atividades, realizando etapas prévias de testes de leitura.

Identificar padrões de erro de resolução e, consequentemente as estratégias utilizadas pelos estudantes, possibilita modificar o planejamento de ensino para corrigir tanto os erros conceituais, quanto os de procedimento (ALMEIDA; PIZANESCHI; DARSIE, 2016; ASHLOCK, 1998; STEFANICH; ROKUSEK, 1992). Problemas no planejamento ou na execução de um programa de ensino adequado são recorrentes, mas descrevem apenas uma das possíveis fontes de erros, conforme já identificado por Bourke (1979).

\subsection{Possíveis fontes de erros}

Além da categorização referente aos tipos de erros cometidos, há também na literatura a descrição de situações ou condições geradoras de erros ou fonte de erros. Ashlock (1998) afirma que, além de avaliar a resposta como certa ou errada, é importante identificar como cada estudante pensa ao resolver um problema, por exemplo. Os conceitos e procedimentos matemáticos que um estudante aprende podem estar corretos ou conter muitos equívocos, uma vez que novos conhecimentos são relacionados a conhecimentos prévios que não são necessariamente corretos.

O autor também afirma que, em algumas situações, procedimentos inadequados produzem respostas corretas e eles podem ser novamente utilizados, aumentando as chances de o estudante cometer erros posteriormente. $\mathrm{O}$ autor também sugere que a aprendizagem de padrões de erro não é resultado intencional das práticas pedagógicas, mas ocorre, sobretudo, quando os estudantes não apresentam os pré-requisitos ou o acompanhamento necessário nas situações de ensino.

Conforme Carmo (2010) destaca, se é possível aprender a errar, é possível também aprender a acertar, uma vez que os princípios de aprendizagem envolvidos são os mesmos. O autor apresenta diversos fatores que podem levar à produção de erros em contexto escolar. Provavelmente os fatores mais destacados no discurso geral referem-se a dificuldades do aluno. Estudantes tanto podem apresentar limitações biológicas, quanto estarem expostos a ambientes que restringem as possibilidades de apresentar comportamentos acadêmicos considerados adequados. Isso, entretanto, não quer dizer que eles não possam aprender. A 
adequação de atividades às limitações e ao ritmo de aprendizagem do estudante é fundamental.

Esse tipo de questão permite a discussão de outras fontes de erros como falhas na programação de ensino, quando o planejamento não é apropriado ou não é aplicado adequadamente, além dos erros causados pela falta de informações, quando há lacunas entre o que o aluno já sabe e o que deve fazer (ASHLOCK, 1998; CARMO, 2010). Outro ponto que deve ser investigado quando há ocorrência de erros é se a linguagem utilizada pelo professor quando falada, ou quando escrita na forma de comandos de questões, por exemplo, é compreendida pelos estudantes (BOURKE, 1979; CARMO, 2010).

Os “esquecimentos” ou a "falta de prática” também são discutidos por Carmo (2010). Argumenta-se que há, pelo menos, duas explicações prováveis para a ocorrência de erros por esses fatores: a repetição mecânica de exercícios que pode não garantir a aprendizagem da lógica necessária para a solução de problemas e outras variáveis, como subprodutos emocionais, que podem interferir no desempenho do estudante. Tais subprodutos podem ser efeitos de concepções inadequadas aprendidas sobre uma disciplina ou conteúdo. Esse tipo de ocorrência não é incomum, uma vez que a Matemática é frequentemente descrita como difícil e não raramente é enunciada socialmente, dentro e fora das escolas, de forma negativa (CARMO et al., 2014).

Outras fontes de erro podem estar relacionadas até mesmo às limitações do professor, que, por diversas razões (como problemas na formação, pouco tempo disponível para planejamento e estudo etc.), pode ter algum tipo de dificuldade com o que se propõe ensinar. Alguns estudos indicam dificuldades com “conteúdos" de Matemática apresentadas por professores dos Anos Iniciais (BORBA; PESSOA; ROCHA, 2012; CARMO; FIGUEIREDO; SILVA, 2000). Muitas vezes, os erros são também induzidos pelos professores. Avaliações que contemplam conteúdos diferentes dos apresentados anteriormente, comandos mal formulados, questões com duplo sentido são exemplos de práticas que podem induzir ao erro (CARMO, 2010).

Ao apresentar diversas condições que podem, isoladamente ou em combinação, levar à produção de erros por parte dos estudantes, destaca-se que o desempenho atribuído como um erro comunica, na maioria das vezes, vários fatores que não apenas uma incapacidade do estudante. Esse tipo de análise permite o questionamento de duas perspectivas que colocam o aluno como responsável por sua aprendizagem: uma delas destaca que erros são inevitáveis e servem de parâmetro para categorização de bom ou mau desempenho, enquanto a outra 
entende o erro como componente fundamental da construção do conhecimento como busca de descobertas individuais do estudante (CARMO, 2010).

Questionar essas perspectivas que centralizam no aluno a responsabilidade de ter aprendido ou não, permite discutir conceitos como ensino e aprendizagem. Ao descrever a relação entre esses processos, Kubo e Botomé (2001, n.p.) afirmam que:

Ensinar define-se por obter aprendizagem do aluno, e não pela intenção (ou objetivo) do professor ou por uma descrição do que ele faz em sala de aula. A relação entre o que o professor faz e a efetiva aprendizagem do aluno é o que, mais apropriadamente, pode ser chamado de ensinar. Nesse sentido, ensinar é o nome da relação entre o que o professor faz e a aprendizagem de um aluno.

Produzir aprendizagem, ou seja, planejar e executar condições que aumentem a probabilidade de o estudante apresentar as respostas consideradas corretas implica descrever operacionalmente o que exatamente espera-se que o estudante faça e quais procedimentos serão utilizados para que o objetivo de ensino seja alcançado. Tão necessário quanto planejar tais condições é também descrever como a avaliação será realizada e que fins ela cumpre.

\section{Método}

\subsection{Bases de dados e descritores}

A busca foi realizada nas bases de dados ERIC, PsycArticles, SciELO e Math Educ Database. Foram selecionadas bases de áreas distintas (Educação, Psicologia, Multidisciplinar e Educação Matemática respectivamente) com o objetivo de ampliar a diversidade dos estudos encontrados. Foram utilizadas as seguintes combinações de descritores: erro $A N D$ matemática; erro $A N D$ procedimento $A N D$ matemática; padrão de erro $A N D$ matemática; análise de erro $A N D$ matemática; e erro sistemático $A N D$ matemática, bem como os correspondentes em inglês e espanhol.

\subsection{Procedimento de busca}

A busca foi delimitada à produção de artigos empíricos e teóricos em português, inglês e espanhol, publicados entre 2012 e 2017 e foi conduzida durante o mês de novembro de 2017 por duas pesquisadoras, de forma independente, a partir das bases de dados e descritores selecionados. Esse procedimento foi orientado para realizar posterior análise de concordância, com o objetivo de diminuir o viés de busca, conforme indicado por Costa e Zoltowski (2014). 
A partir da leitura dos títulos e dos resumos dos trabalhos foram recuperados os artigos que indicassem possíveis variáveis relevantes na produção de erro em Matemática. Resultados duplicados foram excluídos. Posteriormente foram comparados os resultados, identificando-se entre os artigos os que foram igualmente recuperados nas duas buscas independentes, assim como os artigos que não foram igualmente recuperados pelas duas pesquisadoras. Após essa identificação, foi calculada a concordância entre juízes, ou seja, a consistência entre os valores absolutos das classificações das duas buscas (calculada pelo número de vezes em que as juízas concordaram/número total de ocorrências).

De acordo com Costa e Zoltowski (2014), uma possível estratégia a ser adotada, caso um consenso não seja alcançado, é promover uma discussão entre os juízes que realizaram a busca para encontrar um novo consenso. Assim, a avaliação dos artigos diferentemente recuperados entre as juízas foi realizada a partir da leitura conjunta dos títulos e resumos dos trabalhos. Posteriormente, para fins de elegibilidade, os artigos foram lidos na íntegra e excluíram-se aqueles que não atendiam ao objetivo do presente estudo. Por fim, os artigos incluídos foram lidos na íntegra para identificação de características gerais das publicações. A organização das informações retiradas dos estudos foi realizada utilizando software Microsoft Excel $^{\circledR}$.

\section{Resultados}

Inicialmente são apresentados os resultados numéricos de cada etapa da busca realizada e, posteriormente apresenta-se a caracterização dos estudos analisados na íntegra e os principais resultados obtidos.

\subsection{Resultados numéricos da busca}

Foram identificados inicialmente 415 artigos. A partir dos critérios de seleção, a pesquisadora A recuperou 43 artigos e a pesquisadora B, 39. Destes, 35 eram comuns (igualmente recuperados por ambas as pesquisadoras) e havia diferença na seleção de 12 dos trabalhos (concordância absoluta de 70/82 ou aproximadamente $85 \%$ ).

Após discussão dos artigos diferentemente selecionados, foram incluídos 40 trabalhos, dos quais, após leitura na íntegra, cinco foram excluídos por não abordarem possíveis variáveis relacionadas às dificuldades enfrentadas pelos alunos, dois por abordarem concepções de professores ou professores em formação sobre outros assuntos que não os 
erros, um por analisar processamento simbólico e não simbólico e um que não se enquadrava em nenhum dos critérios anteriores, uma vez que apresentava uma crítica ao sistema de provas nacionais para avaliação dos alunos.

Desta forma, 31 artigos (Apêndice A) foram incluídos para análise. A Figura 1 apresenta uma síntese dos dados, desde a identificação inicial, até o processo final de inclusão.

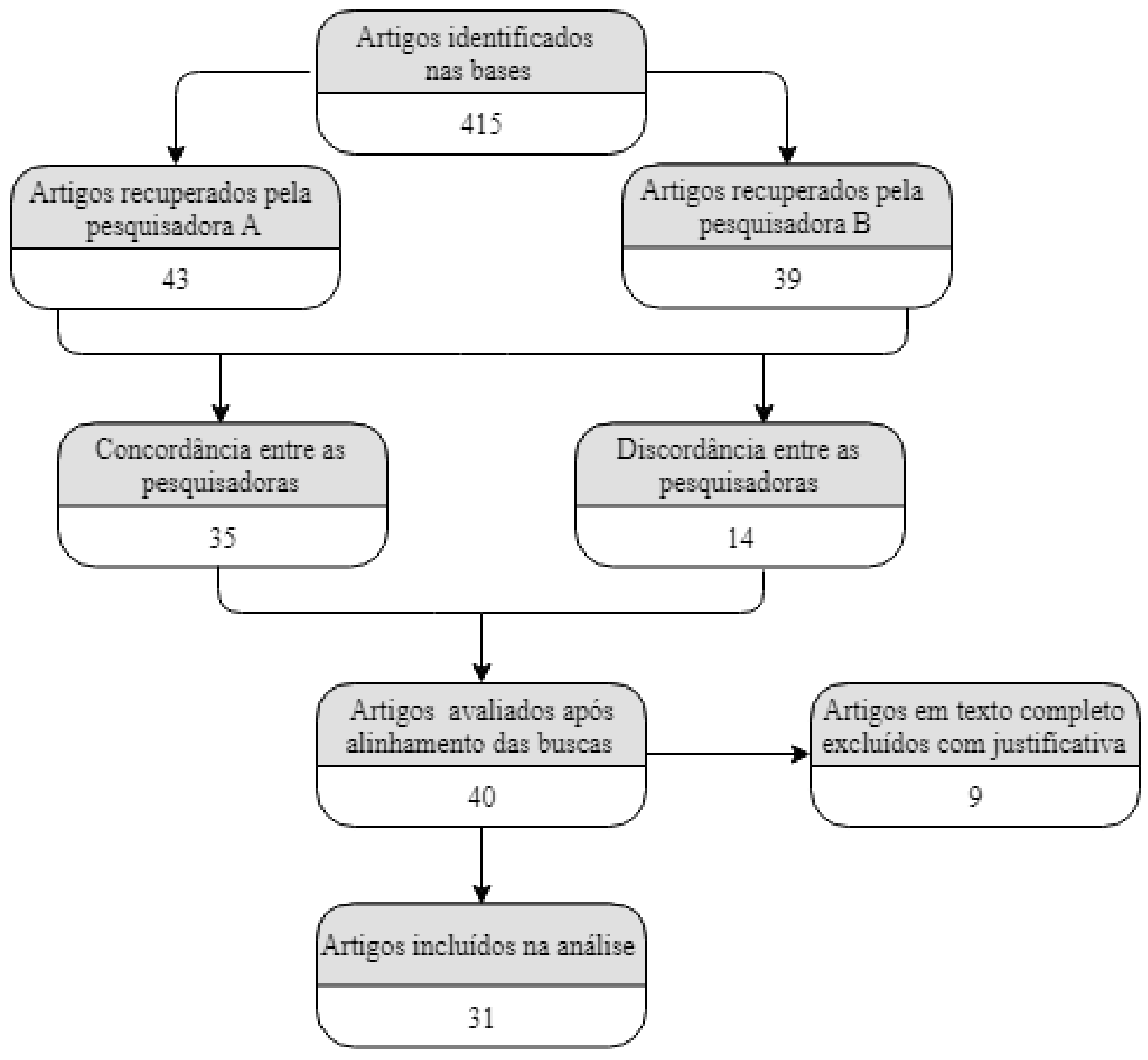

Figura 1 - Dados de identificação, seleção, elegibilidade e inclusão dos artigos buscados. Fonte: Elaborada pelos autores (2019)

\subsection{Caracterização dos estudos incluídos para análise}

A Tabela 1 mostra o número de publicações por ano e país de origem dos artigos analisados na busca 


\begin{tabular}{ccc}
\hline Ano de publicação & Número de artigos & Países \\
\hline 2012 & 3 & Irã (1), Turquia (1), Zimbábue (1) \\
2013 & 5 & Estados Unidos (2), Espanha (1), Austrália (1), Alemanha (1) \\
2014 & 7 & Estados Unidos (2), Reino Unido (1), Brasil (1), Austrália (1), \\
& & Indonésia (1), Canadá (1) \\
2015 & 4 & Líbano (1), Canadá e Austrália (1), França (1), Turquia (1) \\
2016 & 9 & Turquia (3), Arábia Saudita (1), Irã e Estados Unidos (1), \\
& 3 & Brasil (1), Indonésia (2), Quênia (1) \\
2017 & 3 & Arábia Saudita (2), Turquia (1) \\
\hline
\end{tabular}

Tabela 1 - Número de publicações e país de origem dos artigos analisados

Fonte: Elaborada pelos autores (2019).

Considerando o recorte de tempo realizado, observou-se número variável de publicações ao longo dos anos, sendo o maior número de publicações em 2016. Entre 2012 e 2014 houve aumento no número de artigos publicados e, posteriormente houve variação entre aumento e diminuição do número de publicações em relação aos anos anteriores.

Quanto à origem dos estudos, o país de origem das instituições às quais os autores estavam vinculados e que mais publicou foi a Turquia com seis estudos, seguida pelos Estados Unidos com quatro publicações. Em apenas duas das publicações há pesquisadores de universidades brasileiras envolvidos (uma em 2014 e uma em 2016). Foram observadas duas colaborações entre países, sendo elas Canadá e Austrália (em 2015) e Irã e Estados Unidos (em 2016). Quanto ao idioma, 29 dos artigos analisados foram escritos em inglês e dois em português. Não foram recuperados para a análise artigos publicados em espanhol.

O Quadro 1 apresenta os conteúdos abordados nos estudos e a escolarização dos participantes.

\begin{tabular}{|l|l|}
\hline \multicolumn{1}{|c|}{ Conteúdos } & \multicolumn{1}{|c|}{$\begin{array}{c}\text { Escolarização dos } \\
\text { participantes }\end{array}$} \\
\hline Álgebra & \multicolumn{1}{|c|}{ Professores } \\
\hline Álgebra: expressões algébricas, sistemas lineares & Escolares \\
\hline $\begin{array}{l}\text { Álgebra: ordem de operações, equações em uma e duas etapas, sistemas } \\
\text { de equações, desigualdades e polinômios. }\end{array}$ & Escolares \\
\hline Aritmética básica (operações), frações e geometria & Escolares \\
\hline Aritmética, Álgebra e Geometria & Escolares \\
\hline Aritmética: adição e subtração & Escolares \\
\hline Aritmética: adição e subtração & Não descrito \\
\hline Aritmética: multiplicação & Universitários \\
\hline Aritmética: multiplicação & Escolares \\
\hline Aritmética: operações e conceito de reversibilidade & Escolares \\
\hline Aritmética: problemas com dados implícitos & Escolares \\
\hline Aritmética: problemas escritos & Escolares \\
\hline Aritmética: problemas escritos e ditados & Universitários \\
\hline Cálculo & Universitários \\
\hline Cálculo e Geometria & Professores \\
\hline Conteúdo não descrito & Universitários \\
\hline Descrição ampla (conceitos matemáticos) & Universitários \\
\hline Desigualdades (<, >, $\leq \geq)$ & Escolares \\
\hline Equações de 1 ${ }^{\circ}$ grau com uma ou duas operações & Universitários \\
\hline Expressões exponenciais & Universitários \\
\hline Frações: adição, subtração, multiplicação e representação gráfica & \\
\hline
\end{tabular}




\begin{tabular}{|l|c|}
\hline Frações: divisão & Universitários \\
\hline Funções & Escolares \\
\hline Funções e equações quadráticas e gráficos de funções quadráticas & Escolares \\
\hline Geometria: ângulos & Escolares \\
\hline Não descrito & Adultos não escolarizados \\
\hline Números, frações, potência, inverso multiplicativo e raízes & Universitários \\
\hline Permutação e combinação & Escolares \\
\hline Senso numérico & Escolares \\
\hline Teorema de Pitágoras & . \\
\hline
\end{tabular}

Quadro 1 - Conteúdo abordado segundo os autores e escolarização dos participantes nos artigos analisados. Fonte: Elaborado pelos autores (2019).

Dentre os artigos selecionados, somente um se tratava de um estudo teórico. As demais pesquisas eram empíricas e realizadas com escolares do Ensino Fundamental ao Ensino Médio (57\%), universitários (30\%), professores em formação (7\%) e adultos não escolares (3\%). Apenas um artigo (3\%) de pesquisa empírica não explicitou a escolarização dos participantes.

Quanto aos conteúdos abordados nas pesquisas revisadas, há grande variedade de tópicos abordados, com maior frequência em estudos sobre aritmética (35\%) e álgebra (13\%), sobretudo com escolares. A forma de descrever os conteúdos foi também bastante diversificada, uma vez que alguns autores apresentavam os tópicos abordados de maneira mais geral (e.g. funções) e outros mais detalhadamente (e.g. funções e equações quadráticas e gráficos de funções quadráticas). Um estudo não descreveu o conteúdo trabalhado.

Somente um artigo tratava sobre produção de erros em alunos surdos. Um estudo citou a realização de um procedimento para avaliar a presença de comprometimento cognitivo, mas não apresenta os resultados da mesma. Os demais não descreviam/avaliavam as características de desenvolvimento dos participantes, nem a presença ou ausência de deficiências.

Quanto à coleta de informações, dentre as 30 pesquisas empíricas, somente oito (27\%) utilizaram testes padronizados ou adaptados de outros instrumentos padronizados para obtenção de dados e 21 (70\%) desenvolveram instrumentos como provas, questionários e entrevistas para coletar os dados. Em um dos artigos não foi descrito o instrumento utilizado para obtenção de dados.

A Figura 2 apresenta as porcentagens de variáveis relevantes na produção de erros encontradas nos artigos analisados. 


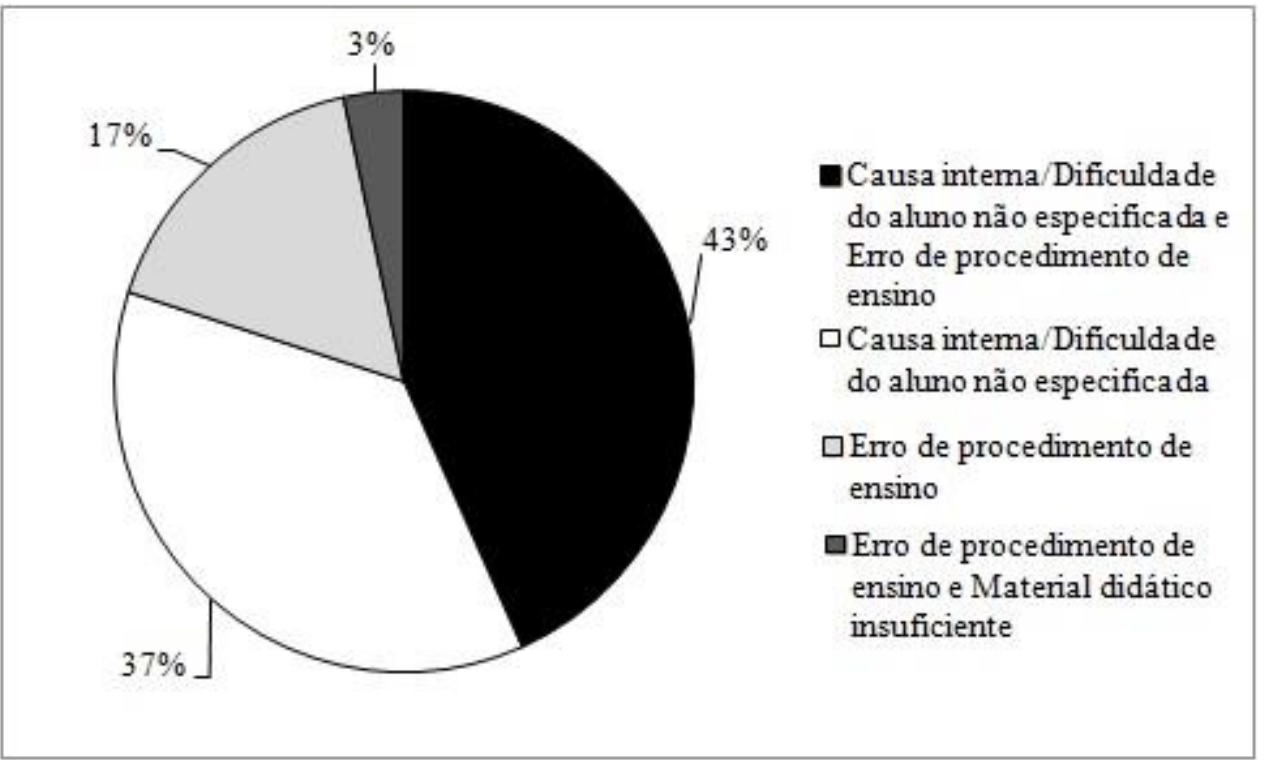

Figura 2 - Variáveis relevantes na produção de erros em Matemática encontradas nos artigos analisados Fonte: Elaborada pelos autores (2019).

Dentre as variáveis que poderiam estar relacionadas com a produção de erros, 11 estudos (37\%) apontaram causa interna ou uma dificuldade do aluno que não foi especificada e cinco (17\%) apontaram erro de procedimento de ensino. Além disso, 14 estudos (47\%) destacaram mais de uma variável relevante na produção de erros, sendo que 13 (43\%) apontaram erro de procedimento de ensino e causa interna ou dificuldade do aluno não especificada, um (3\%) apontou erro de procedimento de ensino e material didático insuficiente.

Cabe ressaltar que nos artigos que apontaram tanto erro de procedimento de ensino quanto causa interna ou dificuldade do aluno não especificada, frequentemente não havia menção sobre questões de ensino até a conclusão do estudo. Em sua maioria, os estudos relataram os erros cometidos, sem relacioná-los a variáveis externas, dando a entender a atribuição a causas internas. Ao final, entretanto, esses estudos sugeriam que algum tipo de mudança de procedimento de ensino poderia auxiliar na correção de erros, atribuindo também aos procedimentos de ensino um papel de variável que produz efeito na emissão de erros por parte dos estudantes.

Em 21 dos artigos empíricos (70\%) os erros foram categorizados. Destes, 18 categorizaram apenas os erros e três todos os tipos de respostas (erros e acertos), embora um destes não tenha apresentado as categorias utilizadas para analisar os erros. As formas de categorizar erros e acertos foram diversificadas. Dos 20 artigos que apresentaram as categorias utilizadas, 15 (75\%) o fizeram por meio de categorias "amplas", a partir das quais eram identificados alguns componentes dos erros, mas não identificavam a estratégia de 
resolução utilizada pelos participantes. Alguns exemplos de categorias amplas são: erros conceituais; respostas parcialmente corretas; erros de cálculo; erros provenientes da falha em aplicar princípios necessários para a resolução do problema; erro de estratégia etc.

Em cinco artigos foram utilizadas categorias "específicas" que apresentavam indícios explícitos do raciocínio dos estudantes. Alguns exemplos de categorias "específicas" são: mover o sinal negativo do expoente para preceder a base; não diminuir do número do qual "pediram emprestado"; realizar a operação sendo o minuendo menor que o subtraendo sem levar isso em conta etc. Ainda, oito artigos (40\% dentre os que utilizaram categorias) descreveram a categorização dos erros com base em estudos prévios da literatura, o que pode indicar algumas tentativas de sistematização de categorias.

Somente sete, entre todos os artigos (23\%), propuseram meios de evitar/lidar com os erros dos alunos de forma detalhada, apresentando sugestões específicas para a prática pedagógica. Outros 15 (48\%) propuseram meios de evitar/lidar com os erros dos alunos de forma geral, sem descrever alterações de procedimentos ou sugestões de práticas específicas que pudessem ser úteis na modificação das respostas. Em nove estudos (29\%) não foi proposta qualquer maneira de evitar/lidar com o erro dos alunos.

Dentre os estudos que apresentaram propostas mais específicas para lidar ou prevenir erros, as sugestões foram diversas. Dos sete artigos que fizeram tais proposições, quatro (58\%) descrevem práticas relacionadas a instruções educativas e procedimentos de ensino. Por exemplo, um deles (ALIBALI et al., 2014) abordou equações de $1^{\circ}$ grau com uma ou duas operações e sugeriu o recurso de criação de histórias escritas pelos estudantes a partir de equações, como uma forma de identificar facilmente os erros de compreensão conceitual do assunto.

Já um estudo que trabalhou com resolução de problemas com dados implícitos apresentou passos para auxiliar a correta resolução por parte dos estudantes, partindo de estratégias simples como sublinhar palavras-chave do problema, construir diagramas etc., além de sugerir que os professores incentivem os estudantes a conferir os resultados, fazendo a resolução "inversa" dos problemas (BARAKÉ; EL-ROUADI; MUSHARRAFIEH, 2015). El-Khateeb (2016) sugeriu, a partir de exemplos, que os professores discutam os símbolos de desigualdades. Houve também a sugestão, no estudo que trabalhou com estudantes surdos, da necessidade de ensinar a diferenciação entre os sinais numerais de uma língua de sinais e o uso dos dedos como objetos para contar, de modo que eles aprendam a utilizar estes dois recursos na hora de operar com numerais (FERNANDEZ-VIADER; FUENTES, 2013).

Além das sugestões relacionadas a instruções e procedimentos, em um estudo (14\%) 
foi apresentada, como possível contribuição à prática docente, o uso de uma ferramenta para auxiliar na identificação de erros conceituais; em outro (14\%) foram sugeridas tarefas e exercícios para a realização de operações básicas, frações e geometria; e um terceiro artigo (14\%) apresentou instruções para aproveitar eventuais erros como oportunidades de ensino. Todas as sugestões, entretanto, não foram avaliadas na íntegra pelos autores de modo a validar sua eficácia.

\section{Discussão}

De acordo com Oliveira (2014), estudos empíricos devem apresentar informações suficientes para que permitam a replicação. A autora destaca que os participantes devem sempre ser caracterizados, bem como os instrumentos utilizados e os procedimentos de coleta e análise de dados. Na presente busca apenas um dos estudos caracterizou efetivamente seus participantes, descrevendo-os como indivíduos com surdez profunda, pré-linguísticos, alfabetizados exclusivamente em língua oral até os oito anos de idade. Os demais estudos não descreveram se os participantes apresentavam desenvolvimento típico ou atípico ou qualquer tipo de deficiência, e apenas um relatou ter realizado alguma avaliação acerca das características de desenvolvimento dos participantes, embora não apresente os resultados da avaliação realizada.

A ausência de informações acerca dos participantes, instrumentos e procedimentos de coleta e tratamento dos dados prejudica a análise da extensão dos resultados obtidos e dificulta a replicação das pesquisas. Além disso, a ausência de descrição detalhada sobre os instrumentos utilizados ou a forma como eles foram desenvolvidos não permite a avaliação, por exemplo, da adequação de linguagem dos enunciados para garantir a compreensão dos estudantes ou da presença de comandos de questões com duplo sentido ou outros aspectos que podem ser relevantes na produção de erros, conforme descrito por Carmo (2010).

Quanto às principais variáveis relacionadas à produção de erros, observaram-se na literatura, principalmente, explicações referentes a causas internas ou dificuldades do estudante não especificadas e a problemas em procedimentos de ensino. Cabe destacar que, em muitos estudos analisados, os autores não deixaram claro quais variáveis consideram importantes na produção de erros.

Entretanto, a partir da conclusão e da sugestão de mudança de procedimentos de ensino que poderiam facilitar o entendimento propostos por estes autores, é possível identificar que eles consideram que o ensino pode não estar sendo suficiente para garantir o 
aprendizado dos estudantes. Além disso, quando os autores descrevem os principais tipos de erros produzidos pelos estudantes (a partir das categorizações) sem relacioná-los a varáveis ambientais, dão a impressão de uma "dificuldade do aluno" que "não compreendeu" ou ainda "não tem maturidade".

Explicações que remetem a causas internas ou dificuldades do estudante também foram identificadas no estudo de Wieman e Welsh (2016). Esses autores aplicaram um questionário com perguntas abertas para investigar as concepções de professores de Matemática e Ciências sobre erros cometidos por estudantes. A maior atribuição de causa de erros dada pelos professores foi referente à categoria "deficiência interna dos alunos", como pouco preparo e ausência de habilidades ou interesse. Poucas respostas foram relacionadas ao tempo e recursos disponíveis limitados e ao grande número de alunos nas salas de aula.

Além de corroborar estudos que atribuem a ocorrência de erros a causas internas ou dificuldades dos alunos, a análise dos estudos revisados confirma também a afirmação de Carmo (2010) de que essas são as fontes de erros comumente encontradas no discurso geral, embora não as únicas. Cabe ressaltar que, mesmo sendo comum, a atribuição às dificuldades individuais dos estudantes é também possivelmente incompleta, uma vez que não há distinção nem entre dificuldades de possível causa biológica, nem pela inserção em ambientes restritivos ao desenvolvimento de repertórios academicamente adequados. Assim, essa "dificuldade do aluno" é apresentada de maneira quase abstrata, sem descrições que permitam compreender como elas se apresentam ou quais as possíveis origens das mesmas. Essa fragilidade de análise pode ser decorrente, inclusive, da identificação muito geral dos participantes dos estudos, conforme já mencionado.

Bourke (1979) enfatiza que muitos alunos reportados como tendo baixo desempenho escolar em Matemática devido à desmotivação e baixas habilidades não apresentam o padrão generalizado de baixo rendimento escolar. Ao considerar que um mesmo desempenho pode ter várias fontes, ou seja, ser influenciado por diversas variáveis, percebe-se o problema em atribuir o erro exclusivamente a uma causa interna ou dificuldade do aluno.

A atribuição da responsabilidade aos estudantes pelos erros cometidos, observada na maioria dos estudos analisados, também distancia a relação entre os processos de ensinar e aprender. Se ensinar for definido pela aprendizagem do aluno e não pela intenção do professor, conforme proposto por Kubo e Botomé (2001), identificar como única fonte do erro uma dificuldade do aluno desconsidera muitas variáveis que podem influenciar o desempenho acadêmico.

Erros cometidos por alunos em Matemática não são simplesmente resultado de sua 
ignorância ou de acidentes situacionais (RADATZ, 1980). Se o aluno não compreendeu, pergunta-se quais as possíveis razões para isso, dentre as quais podem ser citadas a programação e execução de um plano de ensino, possíveis dificuldades do docente, problemas de comunicação entre professor e aluno, a existência de lacunas entre o repertório do estudante e os desempenhos requeridos etc. (CARMO, 2010). Em um estudo para investigar as habilidades de professores em compreender e analisar o pensamento de alunos, Kaplan e Argün (2017) relataram que, ainda que pudessem identificar os erros dos alunos, os professores mostraram-se incapazes de compreendê-los e de atribuir sua origem a outras fontes que não os próprios alunos.

O erro fundamental de atribuição refere-se à tendência de atribuir erros a causas internas do indivíduo, subestimando o conjunto complexo de fatores que estão associados ao comportamento apresentado (ROSS, 1977). Os estudos analisados apontam que os erros têm sido frequentemente associados a limitações internas dos alunos, com poucas descrições que considerem fatores externos, tais como ambiente educacional e qualidade de instrução.

Além da apresentação de variáveis relevantes na produção de erro, observou-se que em $70 \%$ dos estudos analisados foi apresentado algum tipo de categorização dos erros. Essa é uma tendência consolidada na literatura, uma vez que estudos sobre identificação de padrões de erros e categorização de desempenhos são encontrados há várias décadas (ASHLOCK, 1998; BOURKE, 1979; GROSSNICKLE, 1936; ROBERTS, 1968; STEFANICH; ROKUSEK, 1992). Não parece ser uma tendência, entretanto, o uso de procedimentos de correção para os erros identificados nos estudos, conforme realizado por Stefanich e Rokusek (1992).

A categorização dos tipos de erro pode ser um passo importante para a identificação de padrões. De acordo com Ashlock (1998), a identificação de padrões de erro baseada em dados suficientes, a partir de uma análise cuidadosa das práticas de resolução adotadas pelos estudantes, é importante para embasar práticas pedagógicas que possam responder aos procedimentos incorretos eventualmente adotados pelos estudantes.

Observou-se que em $75 \%$ das pesquisas analisadas no presente estudo que realizaram algum tipo de categorização, foram apresentadas categorias amplas, que não descreviam exatamente o que o estudante fazia e tampouco proporcionavam indícios claros de seu raciocínio durante a realização das tarefas. Na presente análise também foi identificada uma tendência geral de descrever erros e categorizá-los sem apresentar muitas oportunidades para facilitar a prática docente.

Essas informações são complementadas por outros resultados do levantamento 
realizado, visto que apenas $23 \%$ dos estudos apresentam recomendações de possíveis práticas pedagógicas que podem ser adotadas diante da produção de erros. Os demais estudos apresentam apenas recomendações genéricas e não sugerem formas detalhadas para lidar com a questão. A ausência de proposições didáticas para prevenir e lidar com erros reflete preocupações que têm sido encontradas recentemente na literatura.

A ausência de interpretação de erros em cursos de formação inicial ou continuada de professores de Matemática foi descrita por Cury (2013), que afirma que discutir os erros e buscar estratégias para superá-los são ações fundamentais na prática dos docentes. Moro, Soares e Spinillo (2017) destacam que há pouca produção sobre ações didáticas que podem auxiliar os professores e futuros professores diante da ocorrência de erros.

Radatz (1980) reitera que a análise de erros é uma oportunidade para diagnosticar dificuldades de aprendizagem e para o desenvolvimento de um ensino de Matemática diferenciado. Esse posicionamento é partilhado por outros pesquisadores (ALMEIDA; PIZANESCHI; DARSIE, 2016; ASHLOCK, 1998) e indica a importância da avaliação dos erros na prática pedagógica. A noção de avaliar a produção dos estudantes enquanto oportunidade, tanto para produzir aprendizagem pelo aluno, quanto para aperfeiçoar a prática docente é discutida por Botomé e Rizzon (1997, p. 7):

\begin{abstract}
Parece necessário desenvolver novos conceitos e novas práticas (procedimentos e tecnologias) de ensino para superar a noção usual de avaliação da aprendizagem fortemente apoiada na noção de "medida de desempenhos" para uma outra em que o papel do professor é sustentado por uma concepção de que avaliar é, continua e imediatamente a cada aprendizagem, dar condições para o aluno corrigir o que realizou ou prosseguir para o novo estágio mais avançado de aprendizagem.
\end{abstract}

\title{
5 Considerações Finais
}

Apesar de a análise de erros produzidos por estudantes em Matemática não ser uma temática recente, o presente estudo sugere que pouca investigação acerca das variáveis que levam ao erro tem sido conduzida. Identificar erros e as possíveis variáveis relacionadas com sua ocorrência é fundamental para que estes, caso aconteçam, sejam utilizados para otimizar os processos de ensino-aprendizagem.

Em contraste, diversos estudos têm apontado a tendência em responsabilizar os alunos por suas falhas, desconsiderando variáveis que têm papel importante no processo de aprendizagem, tais como ambiente educacional, material didático e inclusive práticas pedagógicas. A imprecisão em apontar as variáveis que levam os alunos a cometerem erros também é presente nos artigos analisados, visto que em sua maioria não há sugestões de 
procedimentos claros e explícitos de como evitar ou lidar com os erros produzidos. Esta revisão aponta que ainda há muitos aspectos relevantes na produção de erros que podem ser estudados. Pesquisas futuras devem, por exemplo, atentar para a caracterização dos participantes, para especificação dos instrumentos utilizados, tanto para coletar, como para analisar os erros produzidos de modo a permitir a replicação de estudos. Dado o baixo número de publicações que investigam empiricamente as variáveis que podem estar relacionadas à produção de erros, percebe-se que tal tema pode ser mais amplamente estudado. Outro campo de investigação que pode ser ampliado refere-se à análise de procedimentos de correção a partir de erros identificados.

É importante destacar que, em razão da combinação de descritores utilizada, das bases de dados selecionadas e do recorte de tempo, provavelmente não foram localizados todos os artigos que versaram sobre a temática. Novas revisões devem ser conduzidas para ampliar as análises aqui apresentadas.

\section{Agradecimentos}

Agradecemos à Coordenação de Aperfeiçoamento de Pessoal de Nível Superior CAPES que apoiou financeiramente este trabalho por meio das bolsas de Mestrado e Doutorado fornecidas à primeira e à segunda autora via Edital n 59/2014 - PGPTA.

Agradecemos também à professora Dra. Solange Calcagno pela cuidadosa revisão e contribuições ao trabalho na fase de redação.

\section{Referências}

ALIBALI, M. W. et al. Middle school students' conceptual understanding of equations: Evidence from writing story problems. International Journal of Educational Psychology, Barcelona, v. 3, n. 3, p. 235-264, 2014.

ALMEIDA, D. M.; PIZANESCHI, F. P. M.; DARSIE, M. M. P. O erro no processo de ensino e aprendizagem em Matemática: sua relação com as dificuldades de aprendizagem no contexto escolar. In: ENCONTRO NACIONAL DE EDUCAÇÃO MATEMÁTICA, 2016. São Paulo. Anais... São Paulo: Sociedade Brasileira de Educação Matemática, 2016. p. 1-13. Disponível em: $<$ http://www.sbem.com.br/enem2016/anais/pdf/7480_4035_ID.pdf $>$. Acesso em: 20 maio. 2018

ARTHUR, L. E. Diagnosis of disabilities in arithmetic essentials. The Mathematics Teacher, Reston, v. 43, n. 5, p. 197-202, 1950.

ASHLOCK, R. B. Error patterns in computation: using error patterns to improve instruction. 7. ed. Upper Saddle River: Prentice-Hall, 1998.

BARAKÉ, F.; EL-ROUADI, N.; MUSHARRAFIEH, J. Problem Solving at the Middle School Level: 
A Comparison of Different Strategies. Journal of Education and Learning, Beaver Creek, v. 4, n. 3, p. 62-70, 2015.

BORBA, R.; PESSOA, C.; ROCHA, C. How primary school studentes and teachers reason about combinatorial problems. In: 12 INTERNATIONAL CONGRESS ON MATHEMATICAL EDUCATION, 12., 2012, Seoul. Proceedings... Seoul: Springer, 2016. p. 1795-1802. Disponível em: $<$ https://drive.google.com/file/d/0B3nOb_rG1DUhVV9zY1VEVlRrSTg/view?usp=drive_web\&usp=e mbed_facebook>. Acesso em: 29 maio. 2018

BOTOMÉ, S. P.; RIZZON, L. A. Medida de desempenho ou avaliação da aprendizagem em processo de ensino: práticas usuais e possibilidades de renovação. Chronos, Caxias do Sul, v. 30, n. 1, p. 7-34, 1997.

BOURKE, S. Error patterns in computation. In: 2 AUSTRALIAN ASSOCIATION FOR EDUCATIONAL RESEARCH ANNUAL CONFERENCE, 2., 1979, Melbourne. Proceedings... Deakin: Australian Association for Educational Research, 1979. p. 230-237. Disponível em: https://www.aare.edu.au/data/publications/1979/1979_3_2.pdf

CARMO, J. S. Produção de erros no ensino e na aprendizagem: Implicações para a interação professor-aluno. In: MIZUKAMI, M. G. N.; REALI, A. M. M. R. (Ed.). Aprendizagem profissional da docência: saberes, contextos e práticas. São Carlos: EdUFSCar, 2010. p. 211-227.

CARMO, J. S. et al. Investigando respostas emocionais à Matemática em estudantes de diferentes níveis de ensino. In: VICHI, C. et al. (Ed.). Comportamento em Foco. São Paulo: ABPMC, 2014. v. 3. p. 391-404.

CARMO, J. S.; FIGUEIREDO, R. M. E.; SILVA, L. C. C. Análise descritiva dos comportamentos envolvidos no comportamento de contar. In: CARMO, J. S.; SILVA, L. C. C.; FIGUEIREDO, R. M. E. (Ed.). Dificuldades de aprendizagem no ensino de leitura, escrita e conceitos matemáticos. Belém: Unama, 2000. p. 33-41.

CARVALHO, J. S. F. As noções de erro e fracasso no contexto escolar: algumas considerações preliminares. In: AQUINO, J. G. (Ed.). Erro e fracasso na escola: alternativas teóricas e práticas. São Paulo: Summus, 1997. p. 11-24.

COSTA, A. B.; ZOLTOWSKI, A. P. C. Como escrever um artigo de revisão sistemática. In: KOLLER, S. H.; COUTO, M. C. P. P.; VAN HOHENDORFF, J. (Ed.). Manual de produção científica. Porto Alegre: Penso, 2014. p. 55-70.

CURY, H. N. Uma proposta para inserir a análise de erros em cursos de formação de professores de matemática. Educação Matemática Pesquisa, São Paulo, v. 15, n. 3, p. 547 - 562, 2013.

EL-KHATEEB, M. M. A. Errors analysis of solving linear inequalities among the preparatory year students at king saud university. Journal of Education and Practice, Hong Kong, v. 7, n. 12, p. 124133, 2016.

FERNÁNDEZ-VIADER, M. DEL P.; FUENTES, M. Observando estratégias e buscando soluções: a resolução de operações por adolescentes surdos. Cadernos CEDES, Campinas, v. 33, n. 91, p. 369 386, dez. 2013.

GROSSNICKLE, F. E. Errors and questionable habits of work in long division with a one-figure divisor. The Journal of Educational Research, Bloomington, v. 29, n. 5, p. 355-368, 1936.

KAPLAN, H. A.; ARGÜN, Z. Teachers' diagnostic competences and levels pertaining to students' mathematical thinking: The case of three math teachers in Turkey. Educational Sciences: Theory and Practice, Singapore, v. 17, n. 6, p. 2143-2174, set. 2017. 
KUBO, O. M.; BOTOMÉ, S. P. Ensino-aprendizagem: uma interação entre dois processos comportamentais. Interação em Psicologia, Curitiba, v. 5, n. 1, n.p., 2001.

MORO, M. L. F.; SOARES, M. T. C.; SPINILLO, A. G. Que ações didáticas escolher diante de erros de alunos em problemas matemáticos? Zetetike, Campinas, v. 25, n. 3, p. 418-439, dez. 2017.

MOROZ, M. Leitura e escrita: Avaliando repertórios e detectando dificuldades. In: CARMO, J. S.; RIBEIRO, M. J. F. X. (Ed.). Contribuições da Análise do Comportamento à prática Educacional. Santo André: ESETec, 2012. p. 113-151.

OLIVEIRA, M. Z. Como escrever um artigo empírico. In: KOLLER, S. H.; COUTO, M. C. P. P.; VAN HOHENDORFF, J. (Ed.). Manual de produção científica. Porto Alegre: Penso, 2014. p. 71 - 90.

RADATZ, H. Students' Errors in the Mathematical Learning Process: A Survey. For the Learning of Mathematics, New Westminster, v. 1, n. 1, p. 16-20, 1980.

ROBERTS, G. H. The failure strategies of third grade arithmetic pupils. The Arithmetic Teacher, Reston, v. 15, n. 5, p. 442-446, 1968.

ROSS, L. The Intuitive Psychologist And His Shortcomings: Distortions in the Attribution Process. In: BERKOWITZ, L. (Ed.). Advances in Experimental Social Psychology. [s.l.] Academic Press, 1977. v. 10p. 173-220.

SILVA, A. G. O.; SALVI, R. F.; PASSOS, M. N. O erro matemático interpretado em revistas da área de Educação Matemática. In: VI CONGRESSO INTERNACIONAL DE ENSINO DA MATEMÁTICA, 6., 2013, Canoas. Anais... Canoas: ULBRA, 2013. Disponível em:

$<$ http://www.conferencias.ulbra.br/index.php/ciem/vi/paper/viewFile/1043/273>. Acesso em: 19 maio. 2018

SPINILLO, A. G. et al. O erro no processo de ensino-aprendizagem da matemática: errar é preciso?

Boletim Gepem, Seropédica, n. 64, p. 57-70, 2014.

STEFANICH, G. P.; ROKUSEK, T. An analysis of computational Errors in the use of division algorithms by fourth-grade students. School Science and Mathematics, Hoboken, v. 92, n. 4, p. 201205, 1992.

VON HOHENDORFF, J. Como escrever um artigo de revisão de literatura. In: KOLLER, S. H.; COUTO, M. C. P. P.; VON HOHENDORFF, J. (Ed.). Manual de produção científica. Porto Alegre: Penso, 2014. p. 39-54.

WIEMAN, C.; WELSH, A. The Connection Between Teaching Methods and Attribution Errors. Educational Psychology Review, New York, v. 28, n. 3, p. 645-648, 2016. 


\section{APÊNDICE A - Lista dos artigos incluídos para análise}

ALGHAZO, Y. M.; ALGHAZO, R. Exploring common misconceptions and errors about fractions among college students in Saudi Arabia. International Education Studies, Beaver Creek, v. 10, n. 4, p. $133-140,2017$.

ALIBALI, M. W. et al. Middle school students' conceptual understanding of equations: Evidence from writing story problems. International Journal of Educational Psychology, Barcelona, v. 3, n. 3, p. $235-264,2014$.

ARSLAN, C.; ERBAY, H. N.; GUNER, P. Prospective mathematics teachers' ability to identify mistakes related to angle concept of sixth grade students. European Journal of Education Studies, Bucharest, v. 2, n. 12, p. $190-204,2017$.

BARAKÉ, F.; EL-ROUADI, N.; MUSHARRAFIEH, J. Problem Solving at the Middle School Level: A Comparison of Different Strategies. Journal of Education and Learning, Beaver Creek, v. 4, n. 3, p. $62-70,2015$.

BASIBÜYÜK, K. et al. The mistakes that are made by students with regard to functions: Evidence from erzincan (a province in turkey). Universal Journal of Educational Research, San Jose, v. 4, n. 11 , p. $2523-2532,2016$.

BOOTH, J. L. et al. Persistent and pernicious errors in algebraic problem solving. Journal of Problem Solving, West Lafayette, v. 7, n. 1, p. 10-23, 2014.

CANGELOSI, R. et al. The negative sign and exponential expressions: Unveiling students' persistent errors and misconceptions. The Journal of Mathematical Behavior, Saint Louis, v. 32, n. 1, p. 69 $82,2013$.

DUARTE LEITE, M. et al. A conceptual framework for error remediation with multiple external representations applied to learning objects. Journal of Technology and Science Education, Terrassa, v. 4, n. 3, p. 155 - 166, 2014.

EGODAWATTE, G.; STOILESCU, D. Grade 11 Students' Interconnected Use of Conceptual Knowledge, Procedural Skills, and Strategic Competence in Algebra: A Mixed Method Study of Error Analysis. European Journal of Science and Mathematics Education, Famagusta, v. 3, n. 3, p. 289 $305,2015$.

EL-KHATEEB, M. M. A. Errors analysis of solving linear inequalities among the preparatory year students at king saud university. Journal of Education and Practice, Hong Kong, v. 7, n. 12, p. 124 $133,2016$.

ESTHER YOOK KIN LOONG. Fostering mathematical understanding through physical and virtual manipulatives. Australian Mathematics Teacher, Adelaide, v. 70, n. 4, p. 3-10, 2014.

FERNÁNDEZ-VIADER, M. DEL P.; FUENTES, M. Observando estratégias e buscando soluções: a resolução de operações por adolescentes surdos. Cadernos CEDES, Campinas, v. 33, n. 91, p. 369 386, dez. 2013.

HAGHVERDI, M.; SEMNANI, A. S.; SEIFI, M. The relationship between different kinds of students' errors and the knowledge required to solve mathematics word problems. Bolema: Boletim de Educação Matemática, Rio Claro, v. 26, n. 42B, p. 649 - 666, 2012.

HAGHVERDI, M.; WIEST, L. R. The effect of contextual and conceptual rewording on mathematical problem-solving performance. The Mathematics Educator, Athens, v. 25, n. 1, p. 56- 73, 2016. 
HENKLAIN, M. H. O.; CARMO, J. S.; LOPES JÚNIOR, J. Medidas Comportamentais de Eficácia: Contribuições na Avaliação do Ensino de Operações Aritméticas. Psicologia: Teoria e Pesquisa, Brasília, v. 32, n. 3, p. 1- 9, 2016.

HOLMES, V.-L. et al. Data-driven intervention: Correcting mathematics students' misconceptions, not mistakes. The Mathematics Educator, Athens, v. 23, n. 1, p. 24 - 44, 2013.

ISIK, C.; KAR, T. An error analysis in division problems in fractions posed by pre-service elementary mathematics teachers. Educational Sciences: Theory and Practice, Singapore, v. 12, n. 3, p. 2303 $2309,2012$.

JORDAN, S. Adult science learners' mathematical mistakes: An analysis of responses to computermarked questions. European Journal of Science and Mathematics Education, Famagusta, v. 2, n. 2, p. $63-86,2014$.

LEMAIRE, P.; BRUN, F. Age-related differences in sequential modulations of problem-size and ruleviolation effects during arithmetic problem verification tasks. Memory \& Cognition, New York, v. 44, n. 3, p. $444-453$, abr. 2016.

MAF'ULAH, S.; JUNIATI, D.; SISWONO, T. Y. E. Pupils' error on the concept of reversibility in solving arithmetic problems. Educational Research and Reviews, Nairobi, v. 11, n. 18, p. 1775 1784, 2016.

MAHER, N.; MUIR, T. "I Know You Have to Put Down a Zero, but I'm Not Sure Why": Exploring the Link between Pre-Service Teachers' Content and Pedagogical Content Knowledge. Mathematics Teacher Education and Development, Adelaide, v. 15, n. 1, p. 72 - 87, 2013.

MEMNUN, D. S. et al. Failures and inabilities of high school students about quadratic equations and functions. Journal of Education and Training Studies, Beaverton, v. 3, n. 6, p. 50 - 60, 2015.

MULUNGYE, M. M.; O'CONNOR, M.; NDETHIU, S. Sources of student errors and misconceptions in algebra and effectiveness of classroom practice remediation in machakos county-Kenya. Journal of Education and Practice, Hong Kong, v. 7, n. 10, p. 31 - 33, 2016.

MUZANGWA, J.; CHIFAMBA, P. Analysis of errors and misconceptions in the learning of calculus by undergraduate students. Acta Didactica Napocensia, Cluj-Napoca, v. 5, n. 2, p. 1-10, 2012.

PURNOMO, Y. W. et al. Assessing number sense performance of indonesian elementary school students. International Education Studies, Beaver Creek, v. 7, n. 8, p. 74- 84, 2014.

RACH, S.; UFER, S.; HEINZE, A. Learning from errors: Effects of teachers training on students' attitudes towards and their individual use of errors. PNA, Granada, v. 8, n. 1, p. 21 - 30, 2013.

STAVROU, S. G. Common errors and misconceptions in mathematical proving by education undergraduates. Issues in the Undergraduate Mathematics Preparation of School Teachers, Lubbock, v. 1, n.p., mar. 2014.

SUKORIYANTO, S. et al. Students' errors in solving the permutation and combination problems based on problem solving steps of polya. International Education Studies, Beaver Creek, v. 9, n. 2, p. $11-16,2016$.

ULU, M. Errors made by elementary fourth grade students when modelling word problems and the elimination of those errors through scaffolding. International Electronic Journal of Elementary Education, Oslo, v. 9, n. 3, p. $553-580,2017$.

ULU, M.; AKAR, C. The effect of visuals on non-routine problem solving success and kinds of errors 
made when using visuals. Educational Research and Reviews, Nairobi, v. 11, n. 20, p. 1871 - 1888, 2016.

USMAN, A. I. Geometric error analysis in applied calculus problem solving. European Journal of Science and Mathematics Education, Famagusta, v. 5, n. 2, p. 119 - 133, 2017. 